\title{
Chromospheric Lyman-Alpha Spectro-Polarimeter
}

\section{Ryouhei Kano}

(National Astronomical Observatory of Japan)

R. Ishikawa, N. Narukage, T. Bando,

Y. Katsukawa, M. Kubo, S. Ishikawa (NAOJ),

K. Kobayashi (NASA/MSFC), J. Trujillo Bueno (IAC),

F. Auchère (IAS), with the CLASP team

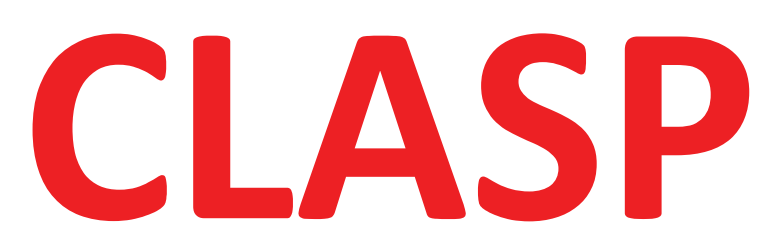




\section{Active Chromosphere}

Quiet Photosphere

We would like to have magnetic-field measurements in low- $\beta$ plasma. 


\section{Chromospheric Lyman-Alpha Spectro-Polarimeter (CLASP)}

A sounding rocket experiment aiming the followings:

- high-precision (0.1\%) measurements of linear polarizations in vacuum-UV (VUV) lights,

- the first measurement of the linear polarization induced by atomic polarization and Hanle effect in the Lyman-alpha line $(121.567 \mathrm{~nm})$, and

- the first exploration of magnetic fields in the upper chromosphere and transition region of the Sun.

The CLASP project was accepted by NASA in 2012, and CLASP will fly with NASA's sounding rocket in 2015! 


\section{International Collaboration in CLASP 12 institutes in 5 countries}

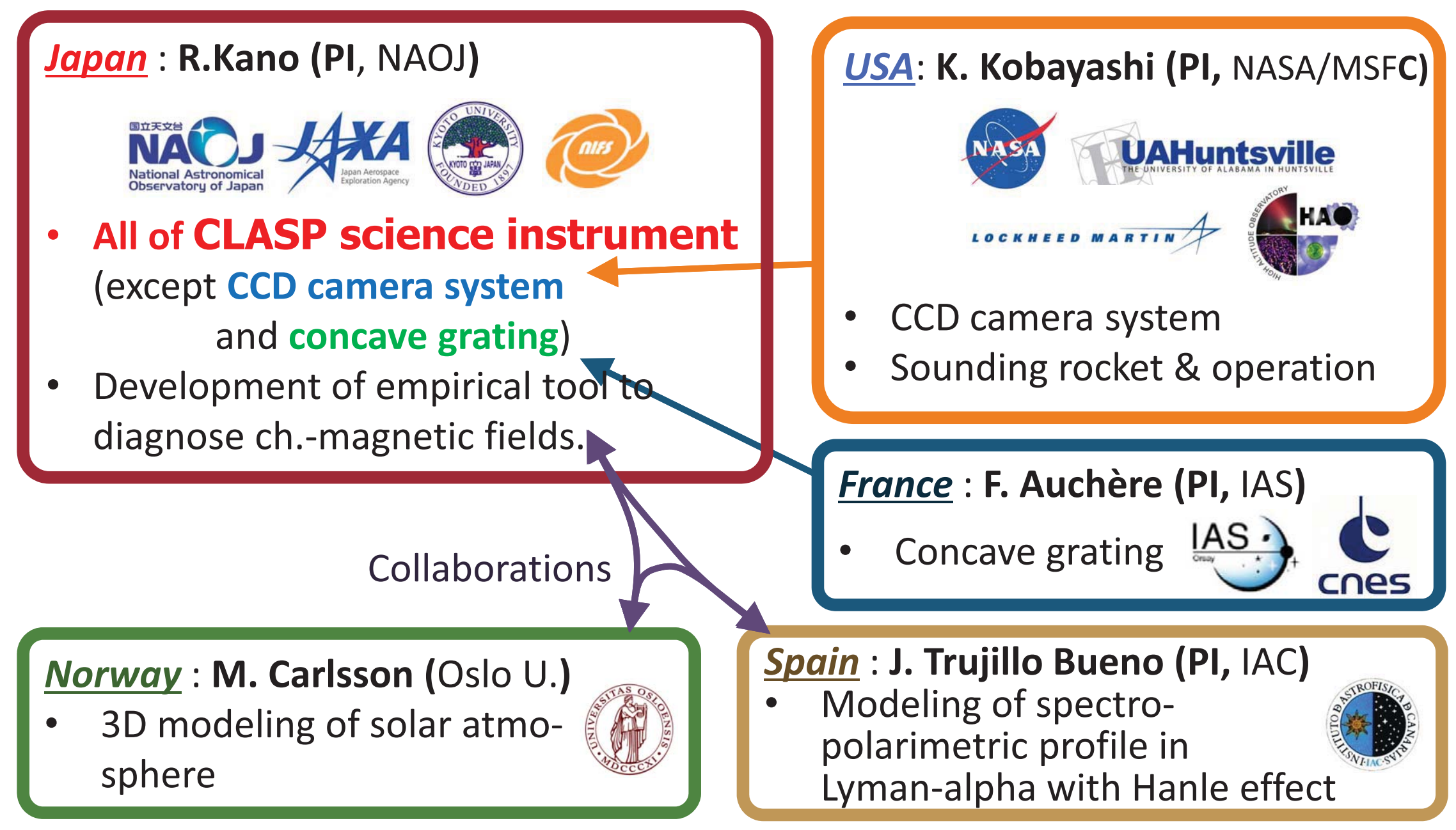




\section{Why Lya line?}

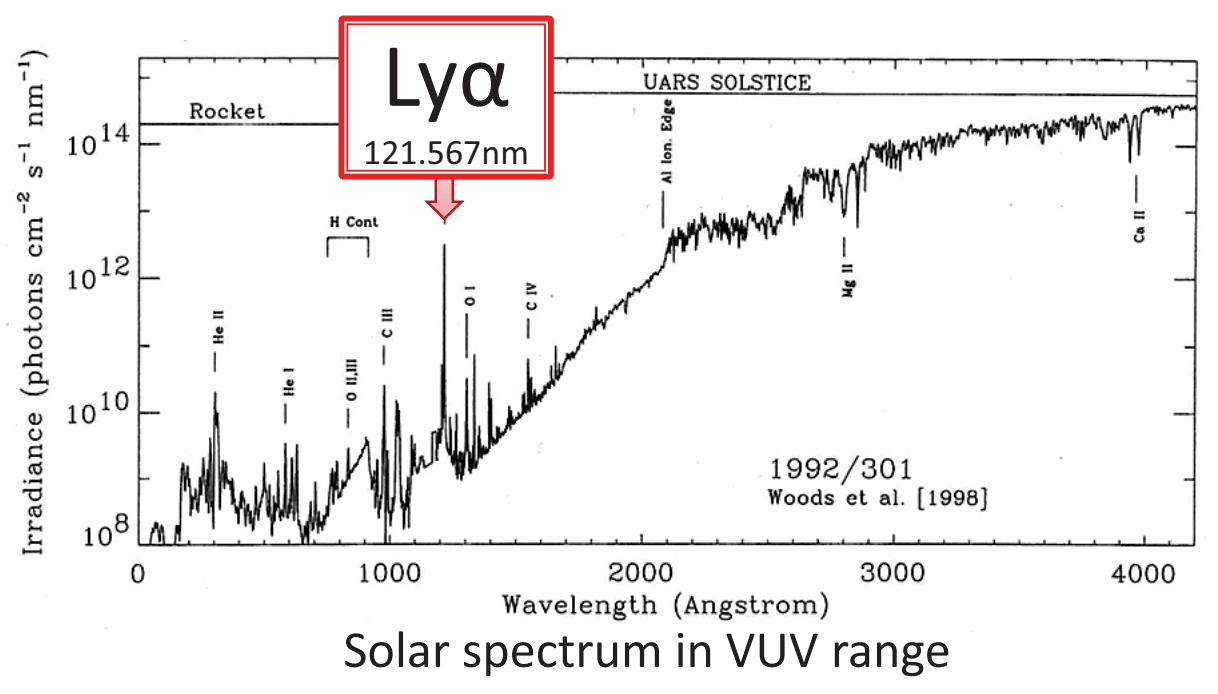

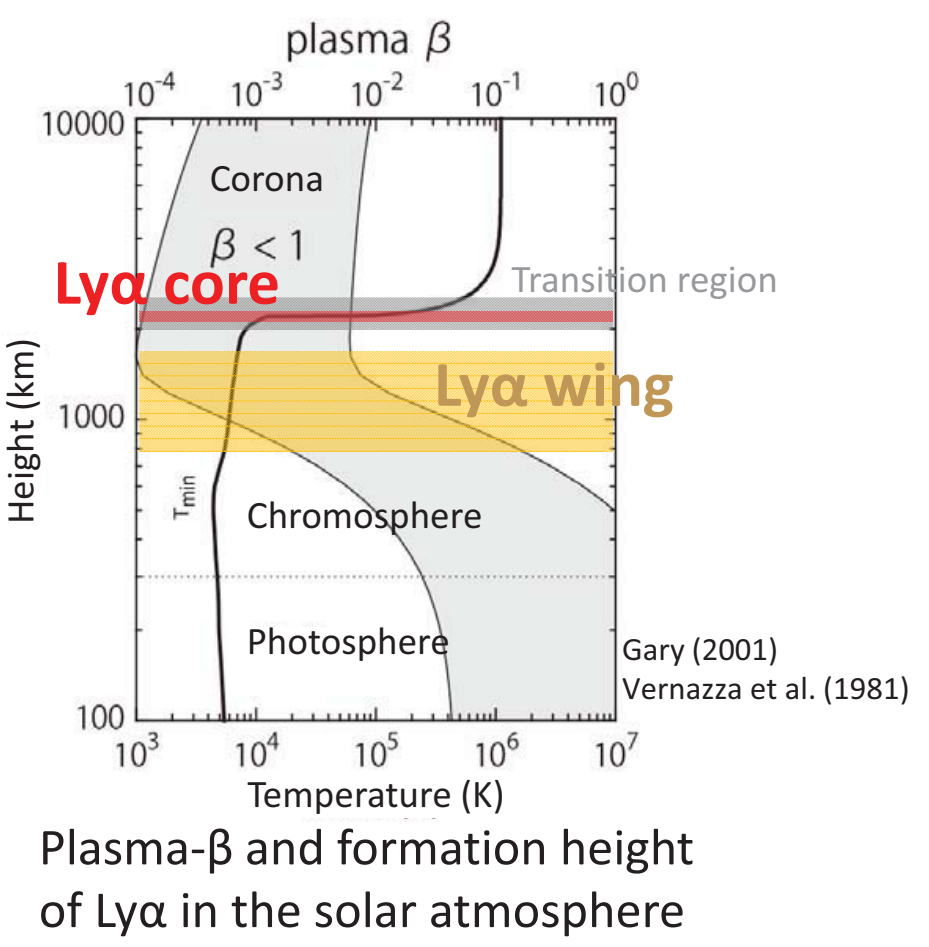

- Brightest line in VUV chromospheric emission lines.

- Bright even in quiet Sun as well as active regions.

- Line core is emitted by the plasma located between higher chromosphere and transition region.

- Good sensitivity to magnetic field of $10-250 \mathrm{G}$ via Hanle effect.

$\rightarrow$ Ly $\alpha$ line is a best candidate to infer magnetic fields in low- $\beta$ plasma $(\beta<1)$ over the entire solar disk. 


\section{Origin of linear polarization in scattered lights}

Step 1: Population imbalance between atomic sublevels induced by anisotropic radiation illuminating atom.

isotropic radiation
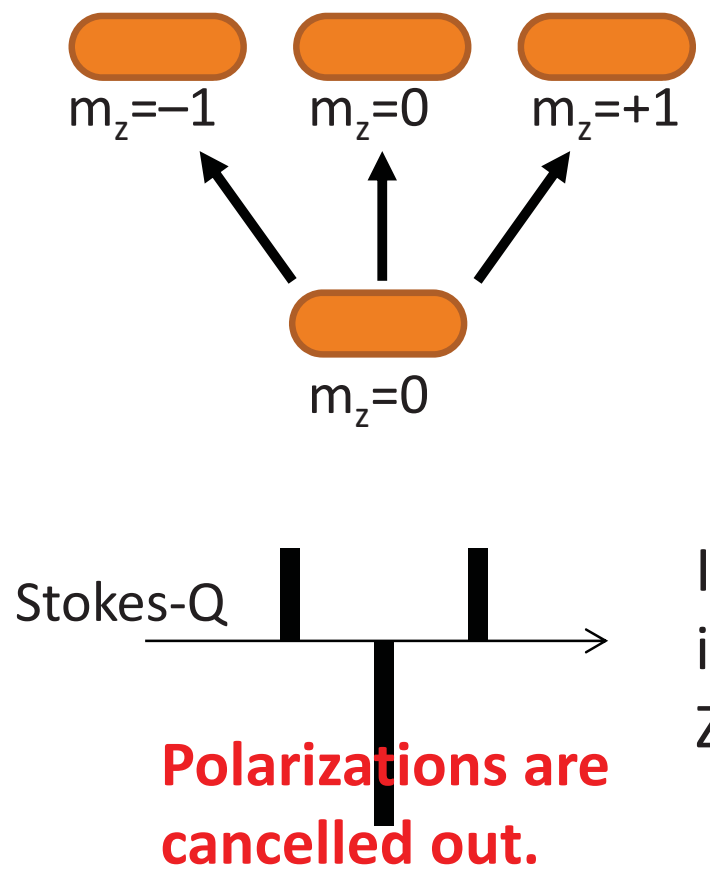

If Doppler width is wider than Zeeman spliting, cancelled out. anisotropic radiation
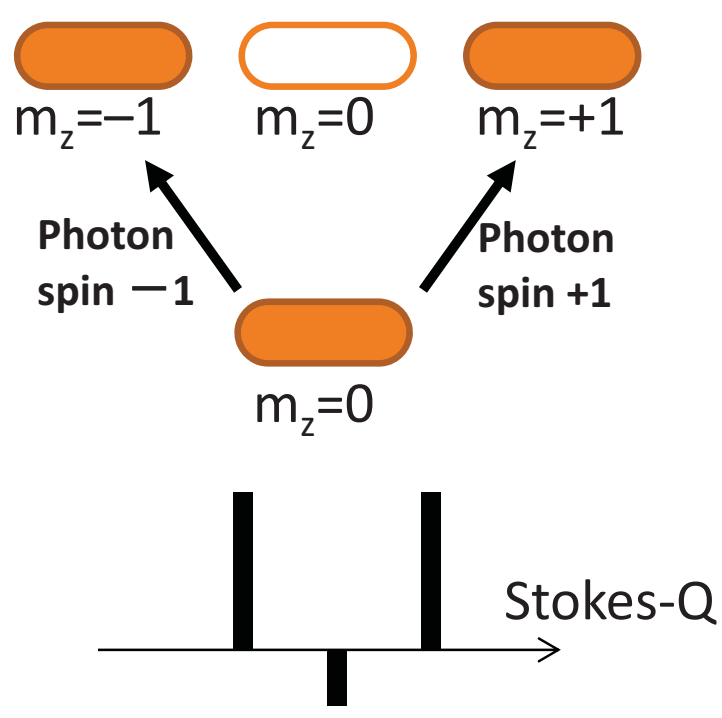

Polarizations remain even after cancellation. 


\section{Origin of linear polarization in scattered lights}

Step2: Quantum coherency by rotation of quantization axes.

Step3: Magnetic fields dephase and decrease the coherence (Hanle effect).

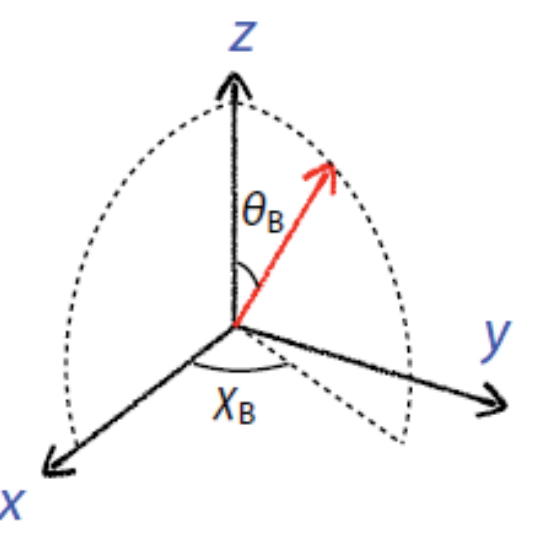
It is a competition between Larmor motion and de-escitation.

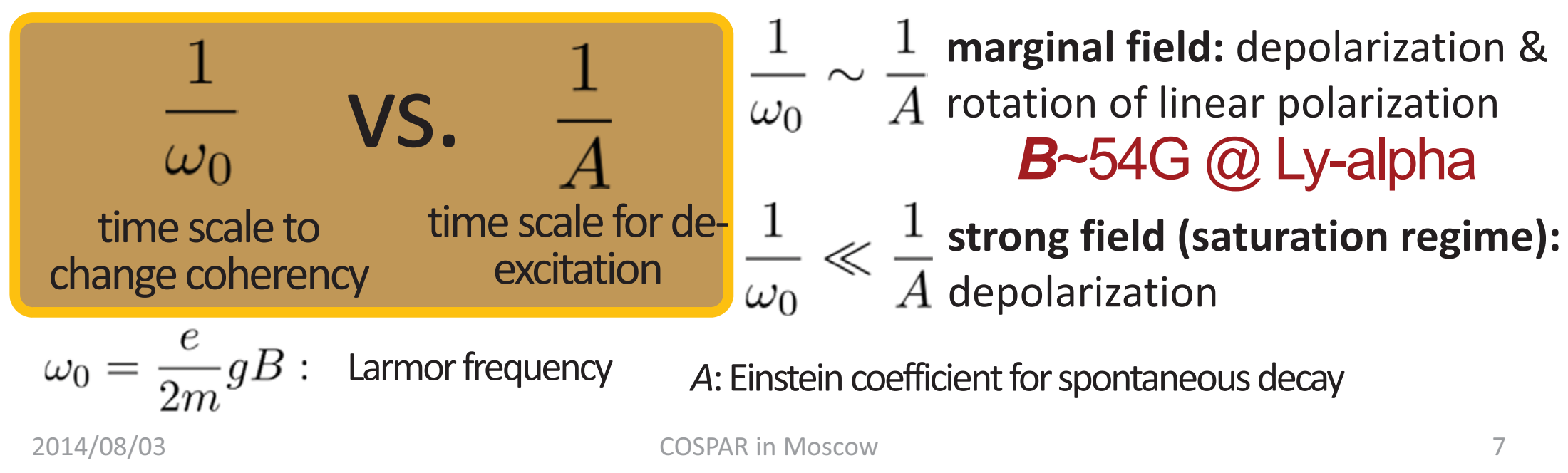




\section{Polarization of}

\section{Hanle effect in Ly $\alpha$}

based on FAL-C model \& CRD scattering (Trujillo Bueno et al. 2011, ApJ)

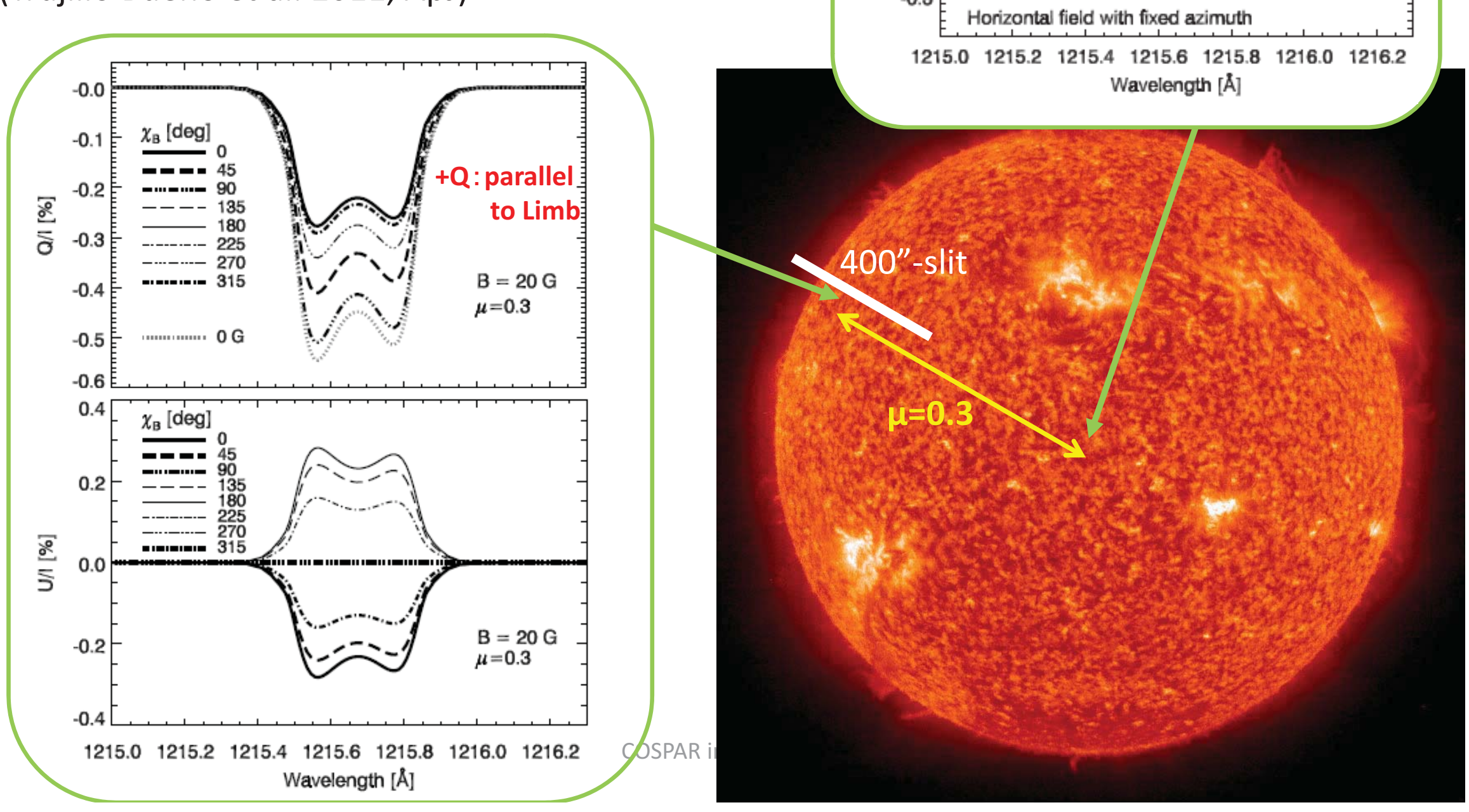




\section{CLASP Instrument: Optics}

Narukage,N. et al. (2014, Applied Optics, in preparation)

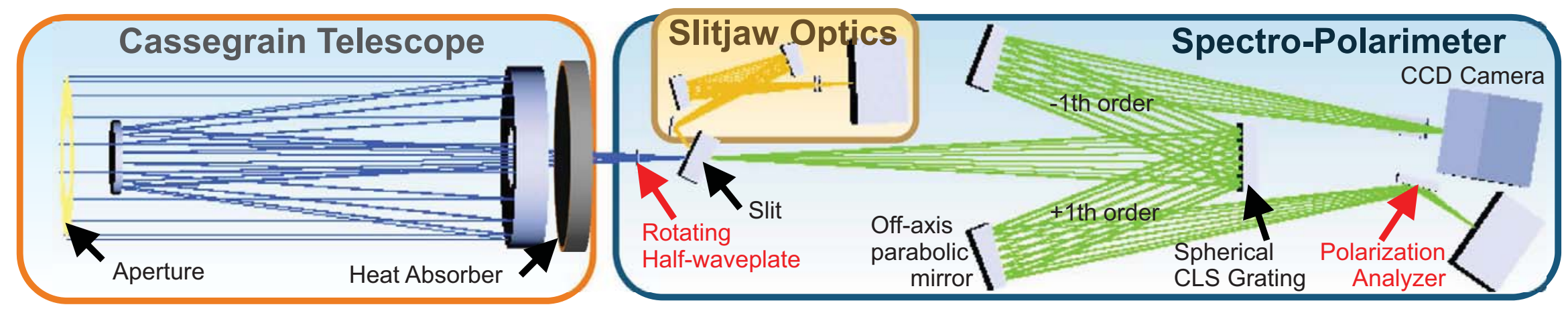

\section{Cassegrain Telescope}

\begin{tabular}{|c|c|}
\hline Aperture & $\phi 270.0 \mathrm{~mm}$ \\
\hline $\begin{array}{l}\text { Effective } \\
\text { Focal Length }\end{array}$ & $2614 \mathrm{~mm}(\mathrm{~F} / 9.68)$ \\
\hline $\begin{array}{l}\text { Visible light } \\
\text { rejection }\end{array}$ & $\begin{array}{l}\text { "Cold Mirror" coating on } \\
\text { primary mirror }\end{array}$ \\
\hline
\end{tabular}

\section{Slitjaw Optics}

\begin{tabular}{l|l}
\hline Wavelength & $121.567 \mathrm{~nm}$ (narrowband filter) \\
\hline Plate scale & $1.03 " /$ pixel \\
\hline FoV & $527^{\prime \prime} \times 527^{\prime \prime}$
\end{tabular}

\section{Spectro-Polarimeter}

\begin{tabular}{|c|c|c|}
\hline Optics & \multicolumn{2}{|c|}{$\begin{array}{l}\text { Dual beam of } \\
\text { Inverse Wadsworth mounting }\end{array}$} \\
\hline Wavelength & \multicolumn{2}{|c|}{$121.567 \pm 0.61 \mathrm{~nm}$} \\
\hline Slit & \multicolumn{2}{|c|}{$1.45^{\prime \prime}$ (width), 400" (length) } \\
\hline Grating & \multicolumn{2}{|c|}{$\begin{array}{l}\text { Spherical constant-line-spacing } \\
3000 \text { lines/mm }\end{array}$} \\
\hline CCD camera & $512 \times 512$ pixel & $13 \mu \mathrm{m} /$ pixel \\
\hline Plate scale & $0.0048 \mathrm{~nm} /$ pixel & 1.11"/pixel \\
\hline Resolution & $0.01 \mathrm{~nm}$ & 3" \\
\hline Sensitivity & $0.1 \%$ & \\
\hline
\end{tabular}




\section{Polarization Measurement}

- CLASP is optimized for linear polarization, because $\mathrm{V} / \mathrm{I}$ is expected to be too small ( 0.005\% @10G in the Ly-alpha by Zeeman effect).

CLASP Polarimeter

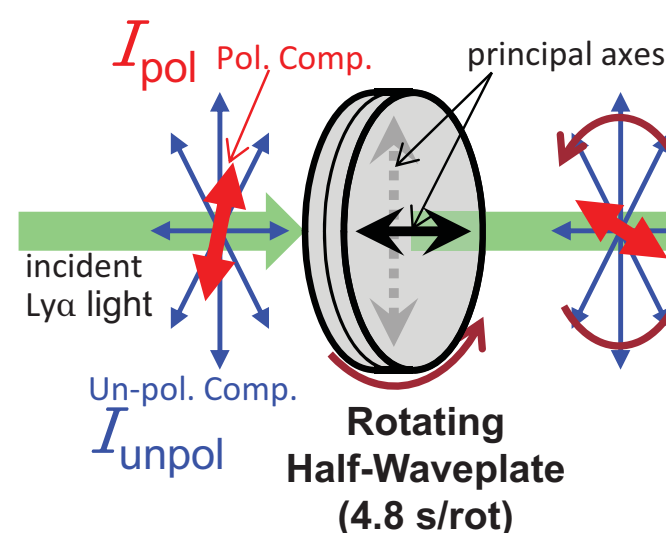

(4.8 s/rot)

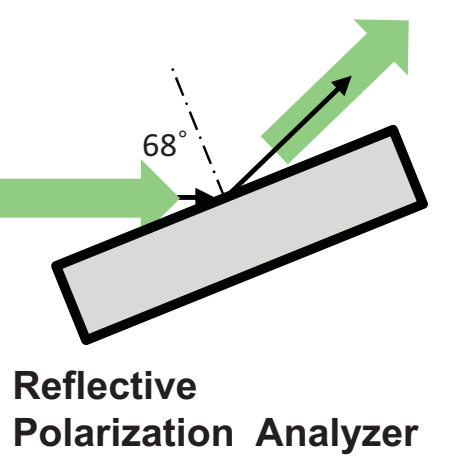

Demodulation from CCD exposures

$$
\begin{aligned}
& Q=\operatorname{aK}\left\{\left(D_{1}-D_{2}-D_{3}+D_{4}\right)+\ldots\right\} \\
& U=\operatorname{aK}\left\{\left(D_{2}-D_{3}-D_{4}+D_{5}\right)+\ldots\right\} \\
& I=\operatorname{Ko}\left\{\left(D_{1}+D_{2}+D_{3}+D_{4}\right)+\ldots\right\}
\end{aligned}
$$

Modulation

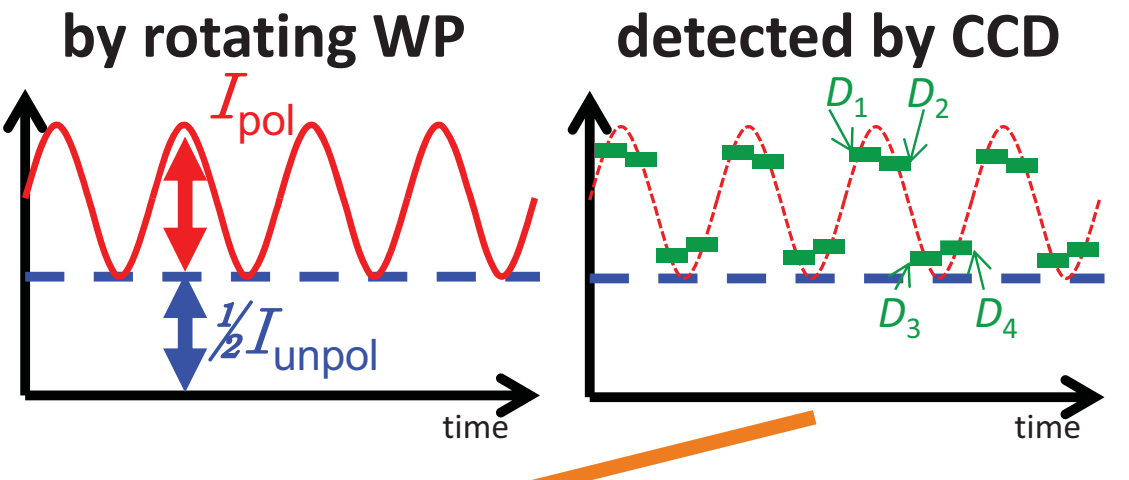




\section{Dual-beam demodulation}

- It will reduce spurious polarizations from time variations.

\begin{tabular}{l|l|l|l|l|l|} 
& $t 1$ & $t 2$ & $t 3$ & $t 4$ & $\ldots$ \\
\hline Ch1 & $1+{ }_{a} Q+a U$ & $1-{ }_{a} Q+a U$ & $I-{ }_{a} Q-a U$ & $I+a Q-a U$ & $\ldots$ \\
\hline Ch2 & $1-{ }_{a} Q-a U$ & $1+{ }_{a} Q-a U$ & $1+{ }_{a} Q+a U$ & $1-a Q+a U$ & $\ldots$
\end{tabular}

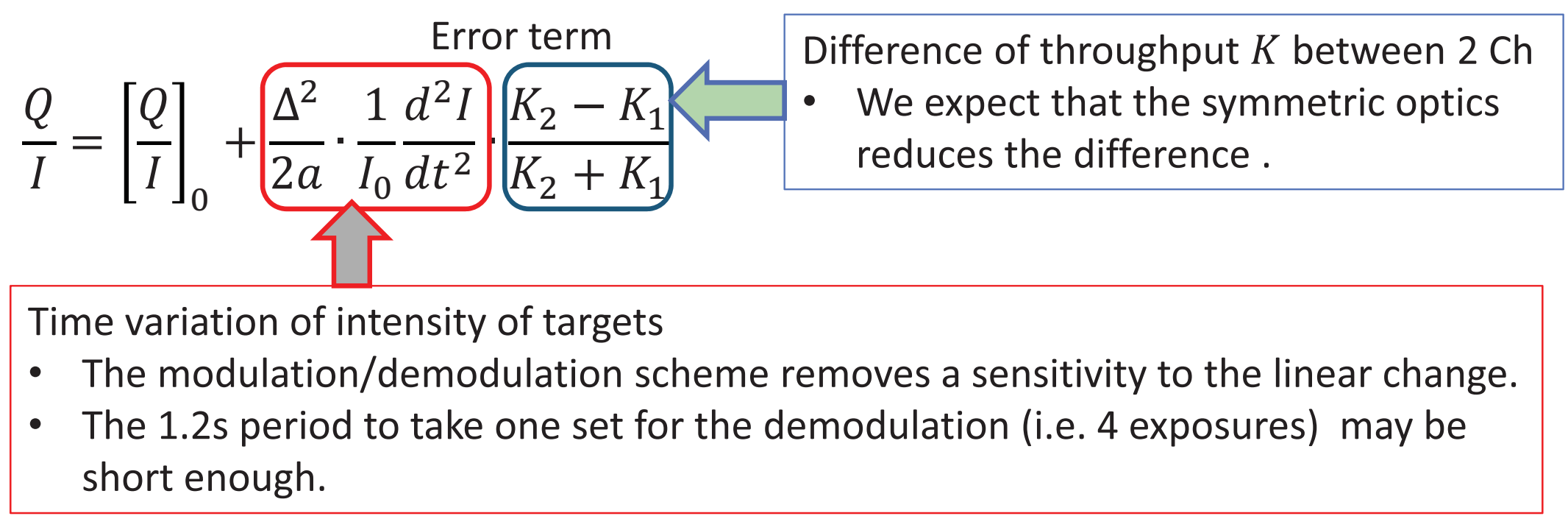

$\Delta$ : exposure interval ( 0.3s) 


\section{Error budget for spurious polarization}

Ishikawa, R., et al. (2014, Solar Physics, in press)

\begin{tabular}{|c|c|c|}
\hline \multicolumn{2}{|r|}{ Cause of error } & error $(1 \sigma)$ \\
\hline \multirow{3}{*}{ 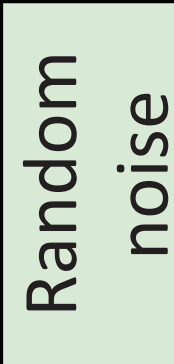 } & $\begin{array}{l}\text { Photon noise at Ly-a center } \\
\text { (10" along slit and } 200 \text { s obs. period ) }\end{array}$ & $0.026 \%$ \\
\hline & Readout noise of CCD cameras & $0.011 \%$ \\
\hline & Fluctuation of exposure durations & $5 \times 10^{-5} \%$ \\
\hline \multirow{3}{*}{$\frac{d I}{d t}$} & Time variation of source intensity & $<0.018 \%+(\sim 0 \%)$ \\
\hline & Intensity variation from pointing jitter & $<0.018 \%+(\sim 0 \%)$ \\
\hline & Image shift from waveplate rotation & $\sim 0 \%$ \\
\hline \multirow{2}{*}{ Tel. } & Off-axis incidence with $200^{\prime \prime}$ & $\sim 10^{-4} \%$ \\
\hline & Non-uniformity of coating on primary & $10^{-3} \%$ \\
\hline SP & Error in polarization calibration & $0.017 \%$ \\
\hline \multicolumn{2}{|r|}{ RSS } & $<0.042 \%(\sim 0.033 \%)$ \\
\hline
\end{tabular}

t: These values are the case for the single channel demodulation, and can be reduced by dual cherannel modulations. 


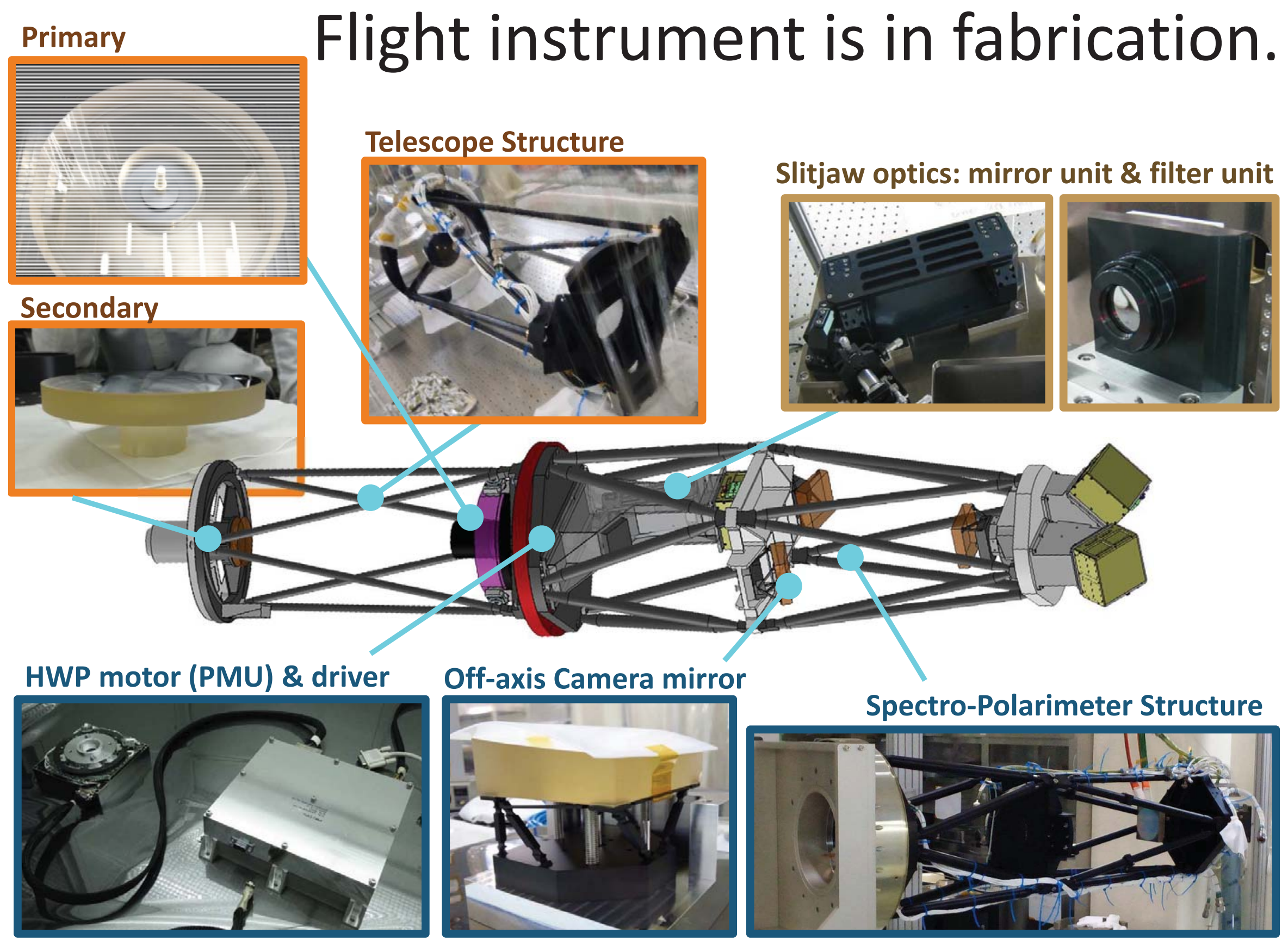




\section{Measurements of flight components are also in progress.}

\section{Half waveplate}

$\mathrm{MgF}_{2}$ WP optimized by Ishikawa, R. et al. (2013, Applied Optics)

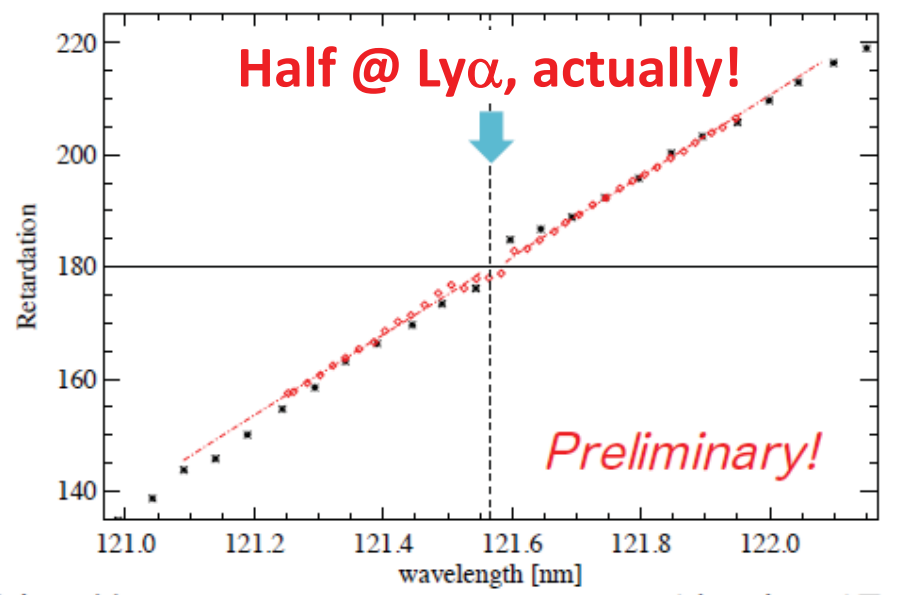

"Cold mirror" coating for primary mirror

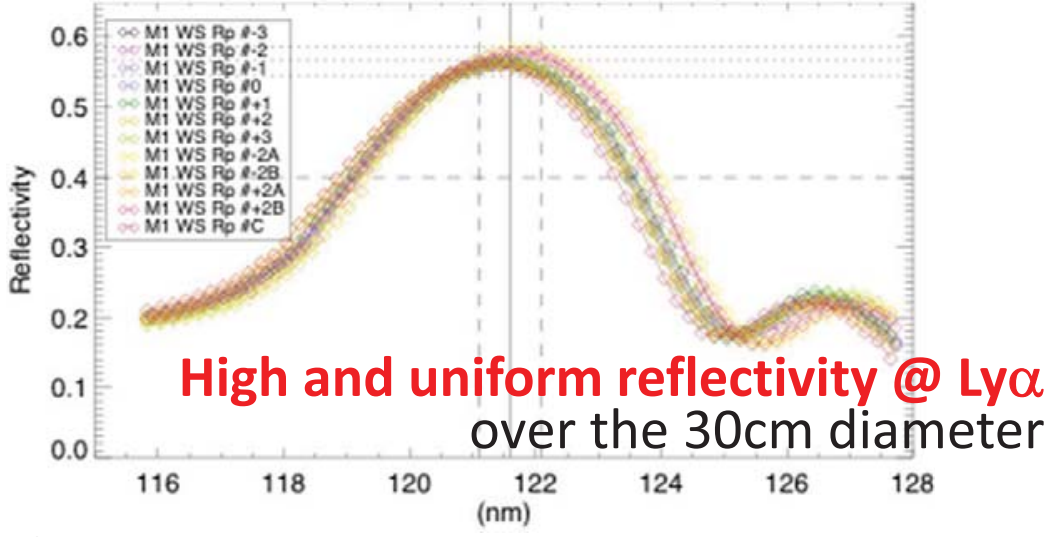

\section{Reflective pol. analyzer}

Multi-layer designed by Bridou et al.(2011. Applied Physics A)

High and uniform pol.-power $=98.9 \%$

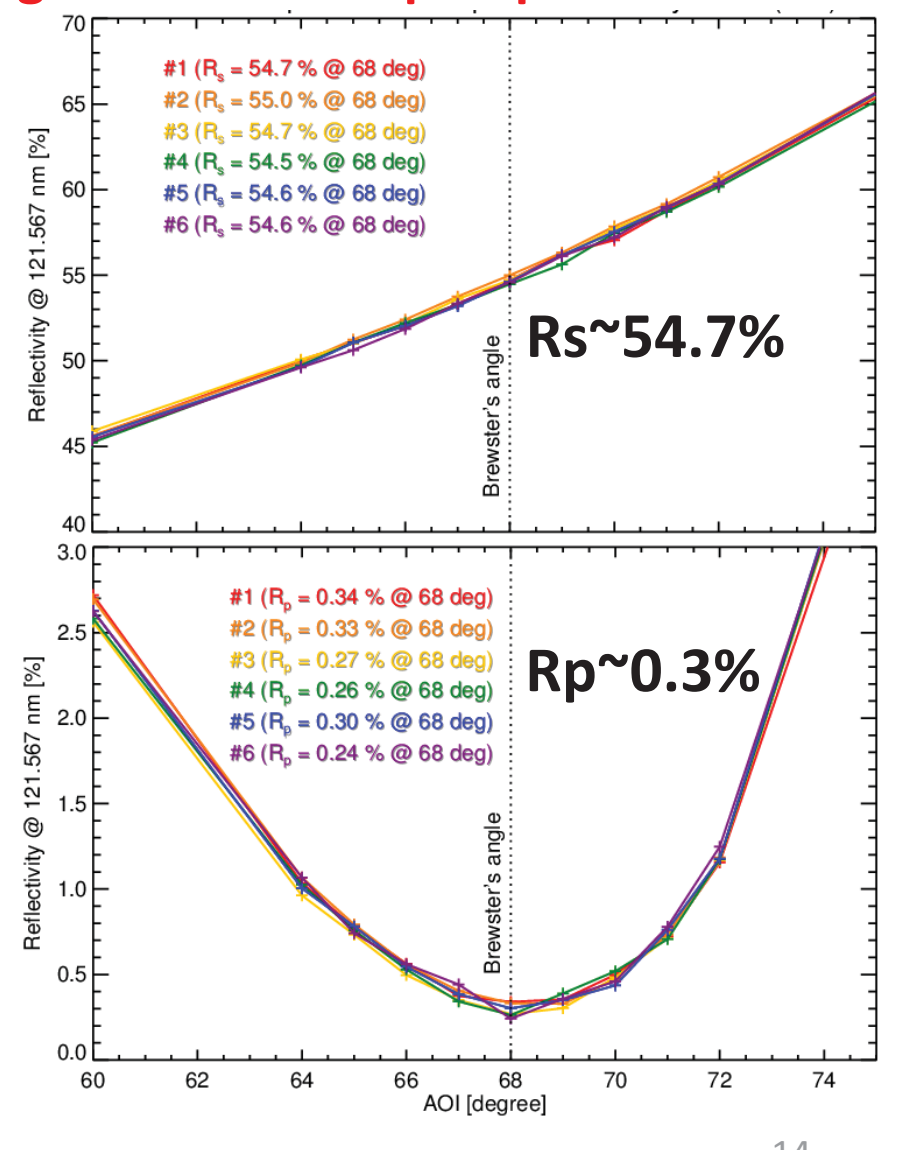

14 


\title{
How ambiguous is $\boldsymbol{B}$-inversion? How to solve the ambiguity?
}

\author{
Ishikawa, R. et al. (2014, ApJ 787, 159)
}

Close-to-limb ( $\mu=0.3)$ obs. of $B=50 G, \theta_{B}=90^{\circ}, X_{B}=120^{\circ}$ Simulated observation

- $\lambda$-res.: $\sigma=0.013 \mathrm{~nm}$

- noise : $\sigma=0.033 \%$

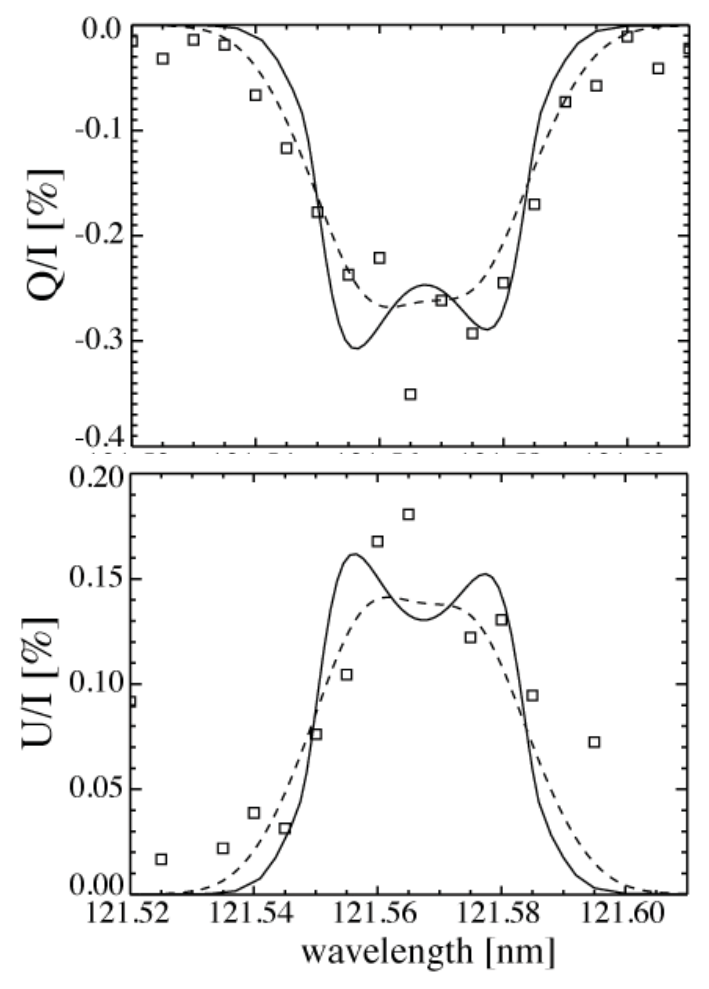

Two regimes:

Take $\chi^{2}$ with all synthetic profiles.

Black dots:

$\Delta \chi^{2} \equiv \chi^{2}-\chi_{\text {min }}^{2} \leq 3.53$,

which corresponds the

confidence level of $63.8 \%$

$(1 \sigma)$ for 3-degree of freedom.
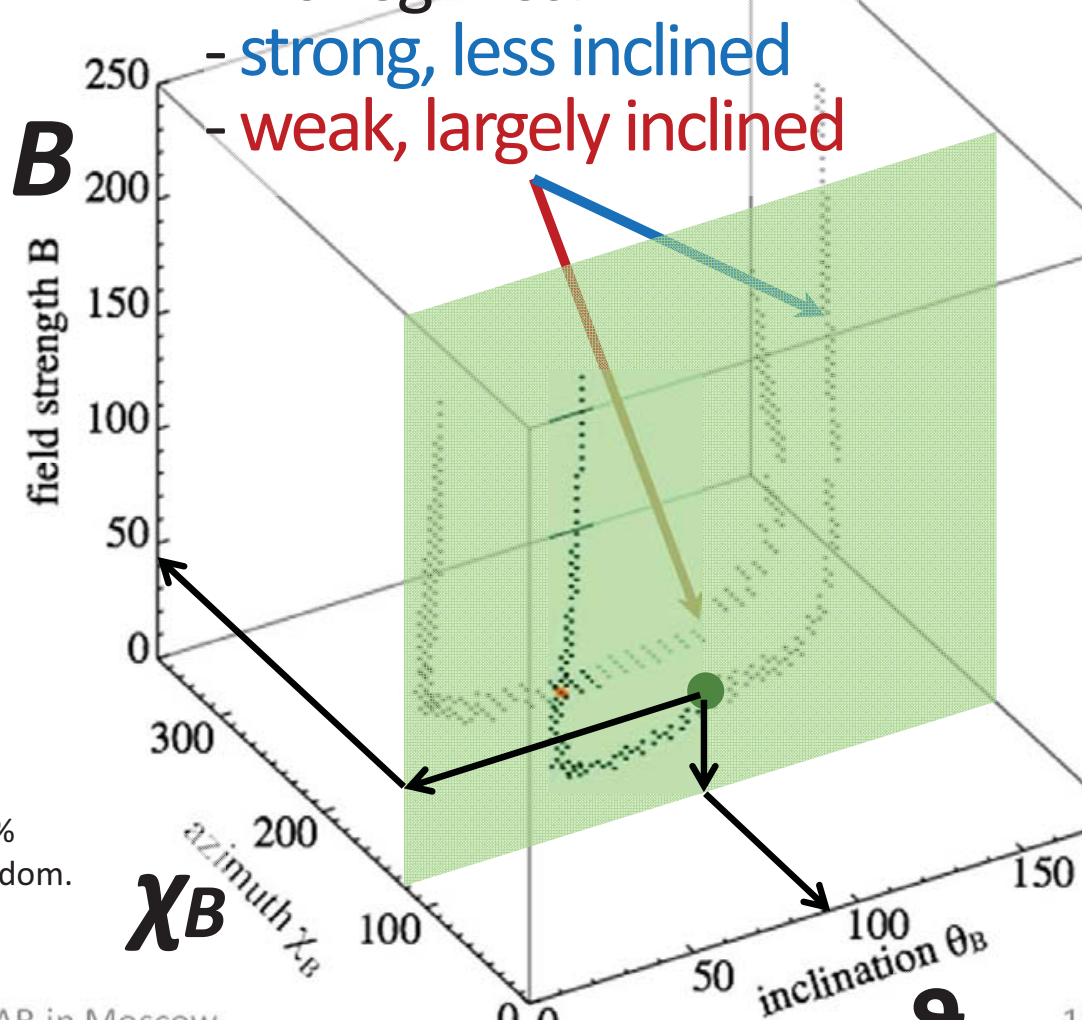

\section{COSPAR in Moscow}

$\vartheta_{B}$ 


\section{Procedure to infer magnetic fields}
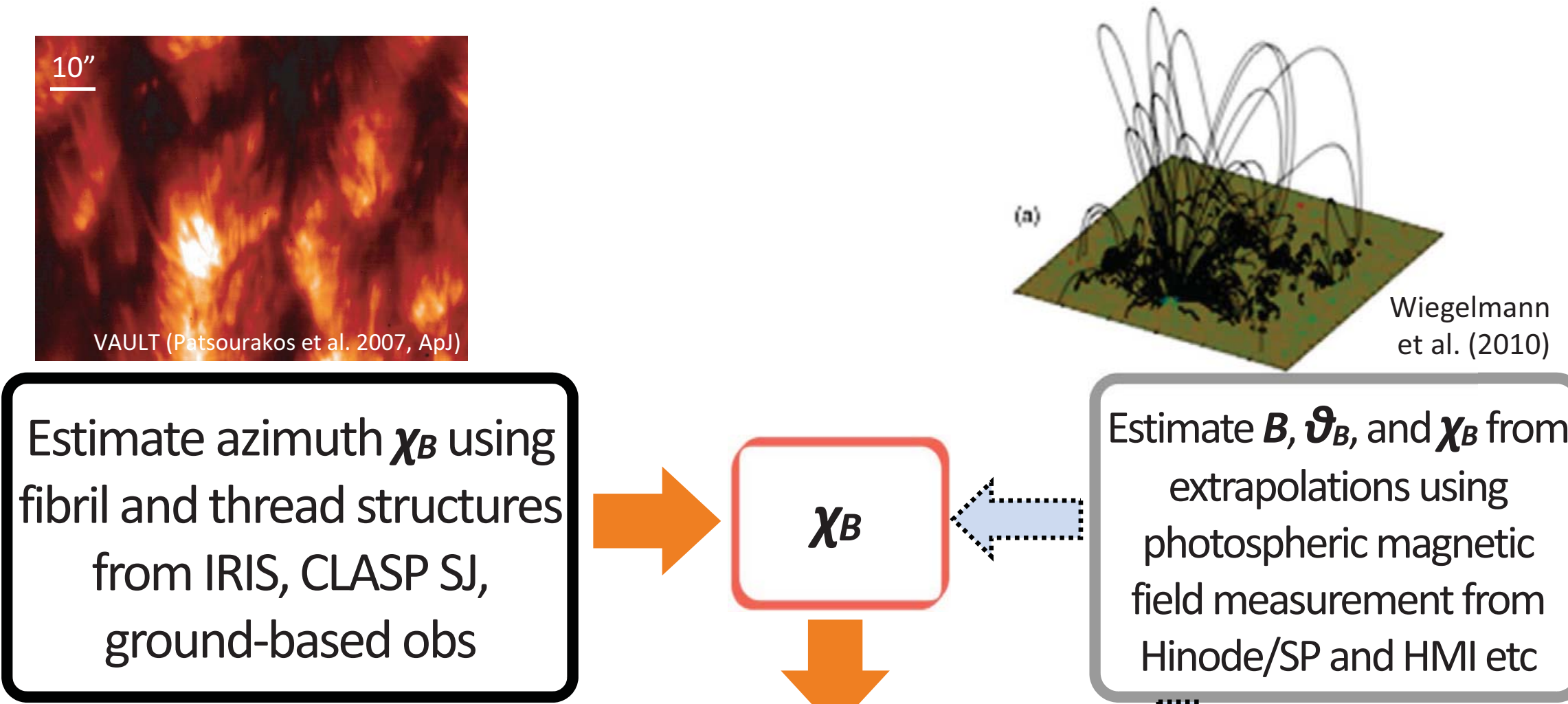

Estimate azimuth $\chi_{B}$ using fibril and thread structures from IRIS, CLASP SJ, ground-based obs 


\section{What will CLASP observe?}

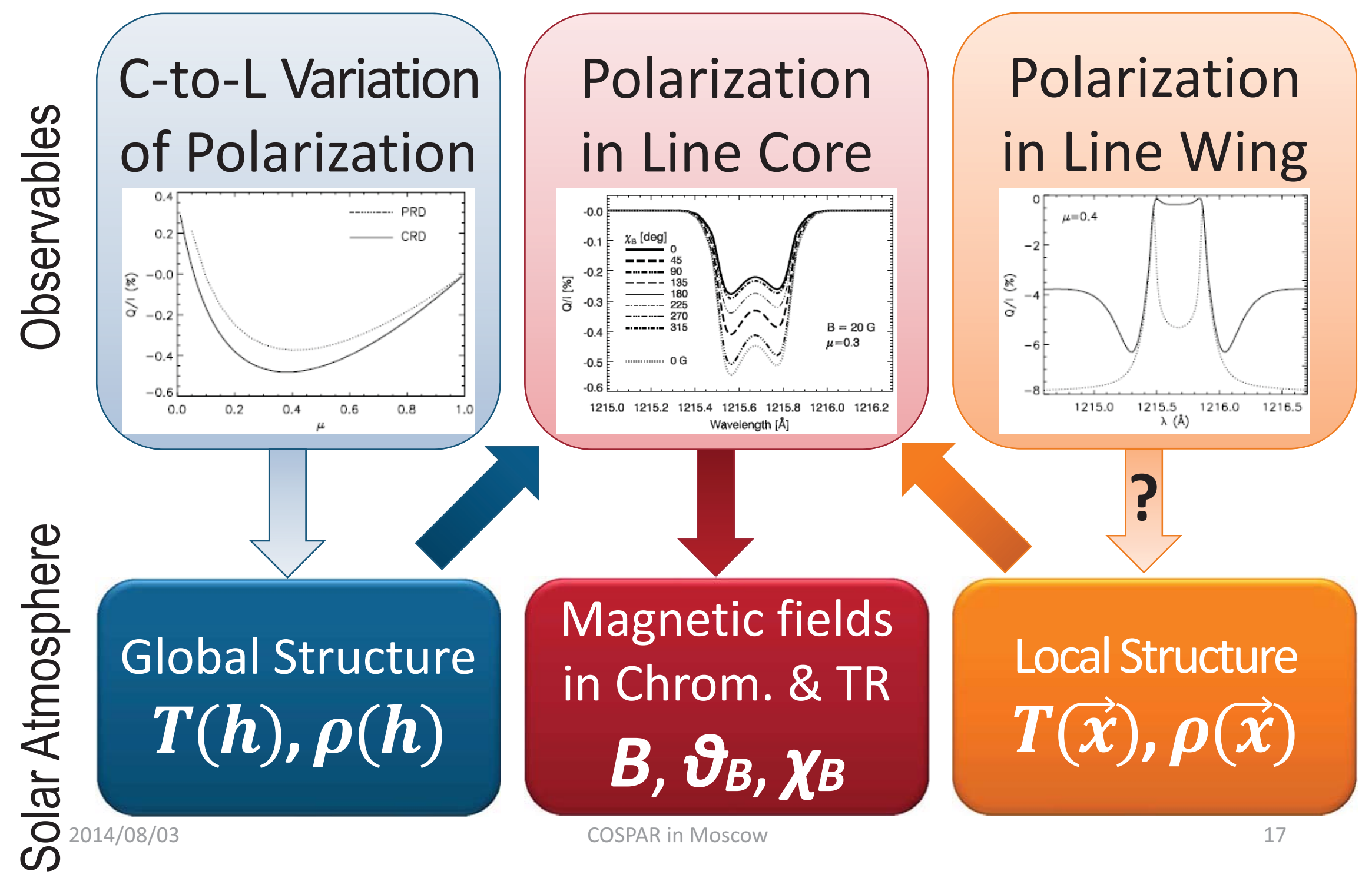




\section{Draft for Coordinated Observation}

IRIS

- during the CLASP flight

- Raster scan of $30^{\prime \prime}$ (scan)x175"(slit)

- Near the limb: $\mu^{\sim} 0.4$ and 0.6 (Scat-pol is maximum at $\mu \sim 0.4$.)

- Mg II h\&k observation.

Hinode/SOT

- before/after the CLASP flight

- Near the limb: $\mu \sim 0.4$.

- Ha imaging \& Photospheric Vector magnetic fields by SP.

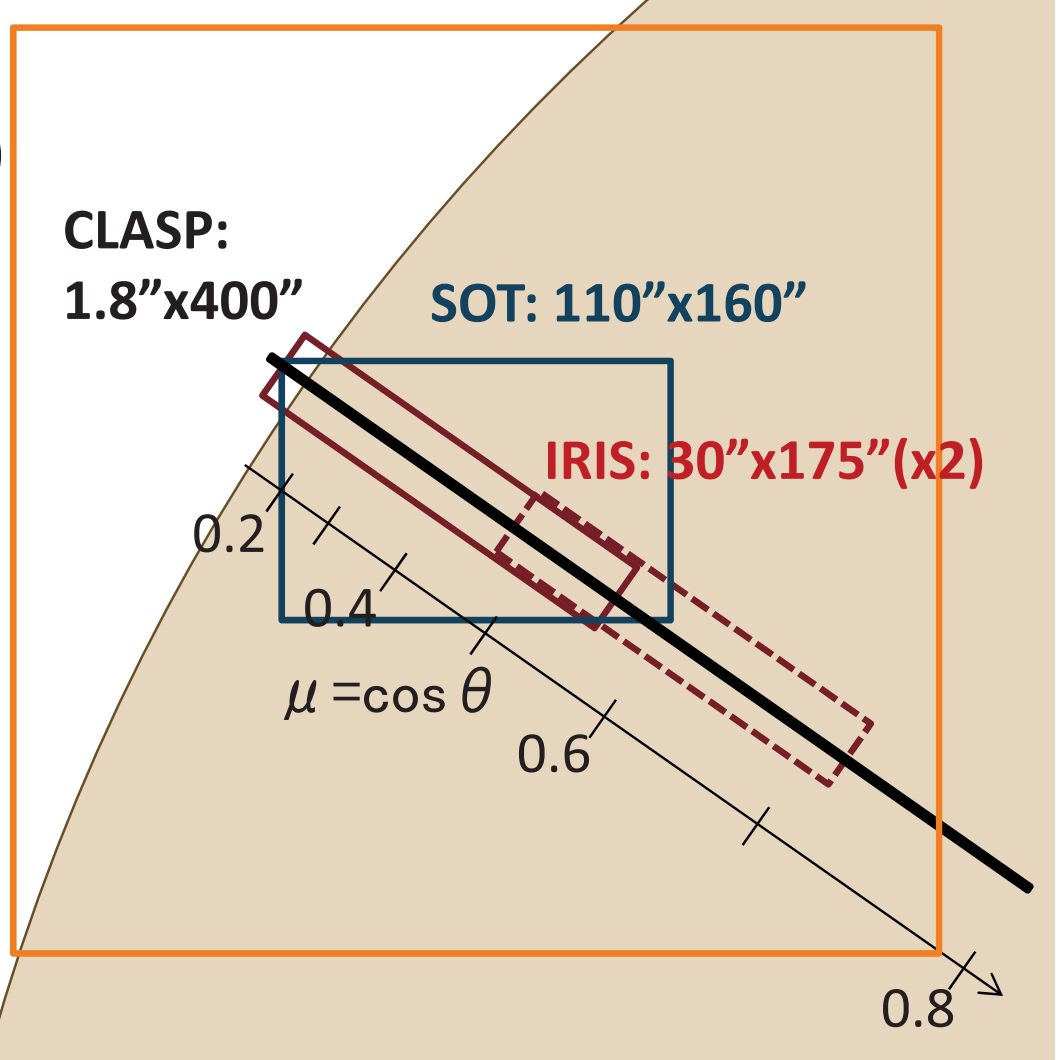




\section{Summary}

- The CLASP project is on-going to infer magnetic fields in upper-chromosphere and transition region.

- The CLASP, a sounding rocket experiment, will be performed in 2015 summer at White Sands in USA.

- Coordinated imaging observations of chromosphere and photospheric magnetic fields are necessary.

- A quick inversion based on plane-parallel atmospheres will be tried at first, but will be followed by precise analysis collaborated with 3D simulations. 